\title{
Prácticas diarias de cuidadoras en centros de desarrollo infantil. Implicaciones para el desarrollo del niño ${ }^{1}$
}

\author{
Marcela Sotomayor Peterson ${ }^{*}$ \\ Macrina Pineda León ${ }^{* *}$
}

\section{Introducción}

Hoy más que nunca la participación de la mujer en la fuerza laboral demanda más y mejores servicios para el cuidado y el desarrollo de los niños. Los centros de desarrollo infantil (CDI) surgen prioritariamente como un derecho de las madres trabajadoras, pero igualmente deben atender los derechos del niño a recibir cuidados y educación de calidad, derechos amparados en marcos normativos internacionales como La Convención de los derechos de los niños y La Educación para todos. En estos, así como en la Constitución Mexicana de 1917 se asienta la obligación del Estado de atender las necesidades de cuidado y fomento al desarrollo de nuestros niños, así como de apoyar a los padres y las familias en sus funciones de crianza (Garbarino y Blasi, 2013).

El reclamo explícito de investigadores como Myers (1992) y Sen (1999) entre otros, acentuó en los últimos años el interés por conocer la calidad con la cual se atienden las necesidades de la infancia y el impacto de los centros de desarrollo infantil para regiones como América Latina (Araujo y López Boo, 2015), y en particular para México (Torres, 2014). En nuestro país, hasta la década pasada eran muy escasos los análisis sobre el tema; casi exclusivamente se podía consultar la información que los organismos oficiales presentaban en sus portales, tal como la encuesta de satisfacción del usuario donde el Instituto Mexicano del Seguro Social (IMSS, 2012) señalaba que el $99 \%$ de los

\footnotetext{
${ }^{1}$ Se usa el término genérico de niño para referirnos igualmente a la niña. * MTC del Departamento de Psicología y Ciencias de la Comunicación de la Universidad de Sonora, msotomayor@psicom.uson.mx

** MHS del Departamento de Psicología y Ciencias de la Comunicación de la Universidad de Sonora, mac_pineda@psicom.uson.mx
}

padres estaban satisfechos con el servicio que recibían en esa guardería.

La muerte de 49 niños en el incendio de la guardería $A B C$ en Sonora, un estado al noroeste de México, el 5 de junio de 2009, hizo evidente la poca información que existía sobre las condiciones en las cuales los CDI's operaban en todos los niveles del país. Entre las acciones ciudadanas, generadas por la toma de conciencia y la indignación social, se dieron ejercicios analíticos y de contraloría social con el apoyo de académicos del estado, liderados por una organización civil en su mayoría integrada por los padres víctimas de la tragedia (Movimiento 5 de Junio).

Este trabajo es resultado de estas acciones e inició con un ejercicio diagnóstico de la situación de la infancia para el estado de Sonora (Sotomayor Peterson y Pineda, 2014).

En su ejercicio diagnóstico, las autoras reportan un vacío de autoridad y opacidad entre las instituciones oficiales responsables de la regulación y operación de los CDI's en Sonora. El estudio señalaba que -pese a que la Secretaría de Educación y Cultura incluye como primer nivel la educación inicial- la institución no reconocía su responsabilidad en el funcionamiento de los CDI's.

Solo los centros ordinarios tanto del IMSS como de otros organismos públicos (Secretaria de Desarrollo Social "SEDESOL", Sistema Estatal para el Desarrollo Integral de la Familia "DIF") que eran directamente operados por ellos, tenían mecanismos de regulación y mantenían estándares adecuados de funcionamiento.

Sin embargo, la cobertura de los centros ordinarios era sumamente limitada. El IMSS en el estado, por ejemplo, reportaba la existencia de 76 CDI's con una capacidad instalada de 14076 lugares y un número de niños inscritos 


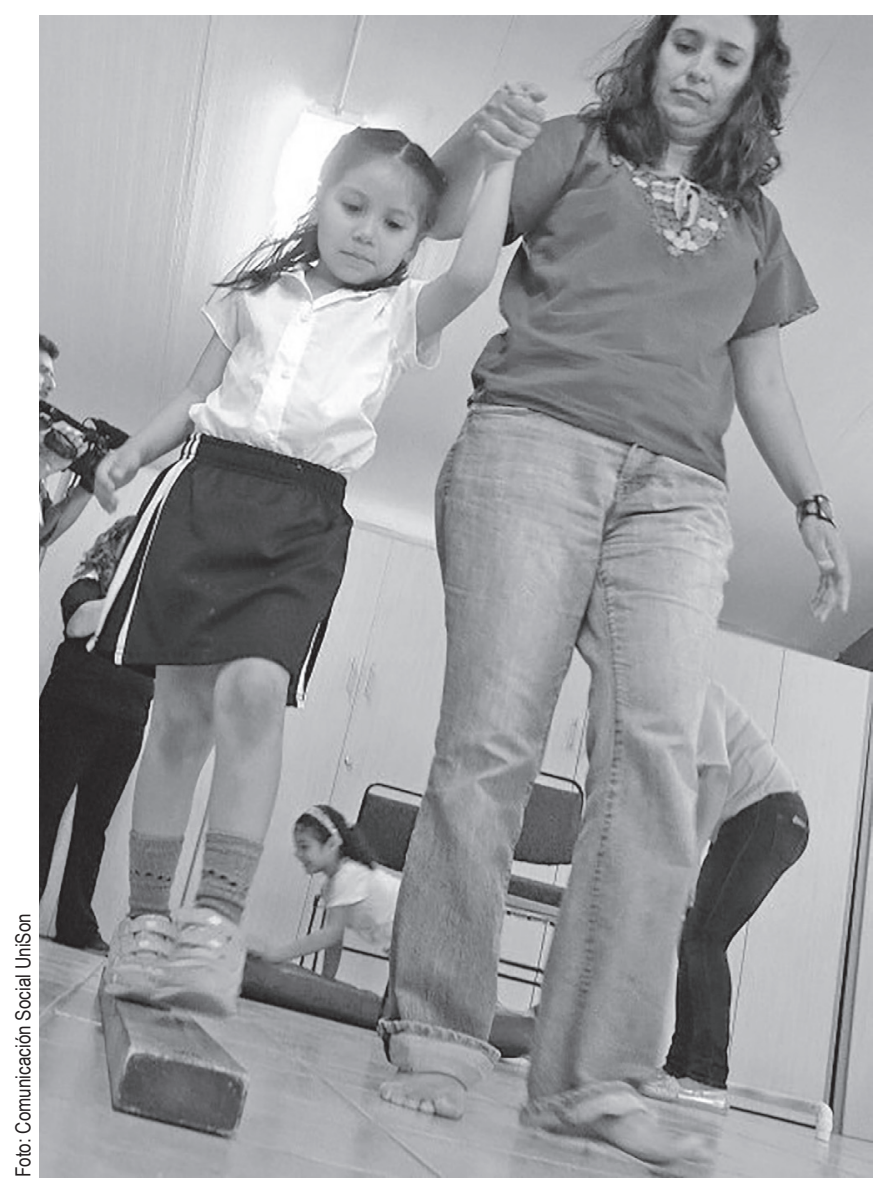

de 12796 para el ciclo 2011-2012; pero solo uno era CDI ordinario, el resto eran subrogados; es decir, atendidos por particulares, los que funcionaban sin apego a las regulaciones existentes, monitoreo, ni evaluación alguna (Sotomayor Peterson y Pineda, 2014).

En otro estudio se añadía que no solo el vacío de autoridad, la poca transparencia, sino las políticas restrictivas en la participación de los padres de familia fomentaban la invisibilidad de los procesos administrativos, las condiciones de funcionalidad y las prácticas al interior de los centros (Nuñez, 2012), sobretodo los subrogados, lo cual se tradujo en riesgos para el bienestar de los niños y sus familias.

La movilización ciudadana incluyó la generación de una nueva Ley general de prestación de servicios para la atención, cuidado y desarrollo integral infantil (Movimiento 5 de Junio) aprobada en el Boletín Oficial del 24 de octubre de 2011 que, evidenciando las fallas de la normatividad anterior, proponía mecanismos que las subsanaran.

\section{El impacto del CDI en el bienestar y desarrollo del niño}

La Encuesta nacional de empleo y seguridad social, ENESS 2013 (INEGI, 2014) reporta que el $53 \%$ de los mexicanos afiliados a un organismo de seguridad social son mujeres; el $40 \%$ de estas son las titulares de la afiliación. El $15 \%$ de los niños mexicanos menores de seis años, cuyas madres trabajan fuera del hogar, asisten a una guardería o CDI; el $76 \%$ de ellos permanece entre cuatro y hasta ocho horas diarias. De los que no asisten a CDI, el $61 \%$ está bajo el cuidado de su abuela; y el resto es cuidado por otra persona.

Entre las razones reportadas para no asistir a un CDI están la falta del recurso económico para pagarlo (15.9\%) y la desconfianza en el buen servicio (15.5\%); el resto argumenta otras razones (no lo necesita, prefiere no comentar, etc.) (Instituto Nacional de Estadística y Geografía "INEGI", 2014).

Estos datos son importantes porque ofrecen indicadores con representación nacional y estatal que evidencian sobretodo el poco acceso que se tiene a los servicios de CDI. Ante el no acceso al servicio público y la dificultad económica para pagar el servicio privado, la gran mayoría opta por dejar el cuidado de sus hijos con familiares u otras personas. Es innegable que una familia con las condiciones materiales, sociales y afectivas adecuadas para satisfacer las necesidades de los niños es el lugar óptimo para su desarrollo (Grusec, 2002); pero en familias que enfrentan limitaciones para ejercer el rol parental se pone en riesgo el desarrollo de sus niños.

Lamentablemente, en las condiciones actuales de nuestro país, con índices crecientes de pobreza y baja calidad de vida, numerosas familias enfrentan estrés familiar severo (horarios extenuantes de trabajo, bajos ingresos, pocos recursos para la estimulación del niño, etc.) fomentándose así descuido en las prácticas de crianza, entre otras (Gómez, Muñoz y Haz, 2007).

Los niños menores de cinco años sufren con mayor peso las consecuencias de la privación material, social y afectiva (Carrion, 2010; Tierney y Nelson, 2010). La falta de estimulación adecuada en el hogar pone en desventaja el desarrollo cognitivo y predice un bajo nivel de rendimiento académico del niño, dibujándose así la ruta hacia la exclusión del niño (Harper, Alder y Pereznieto, 2012). Por esta razón, el acceso a un CDI de calidad es una estrategia fundamental para reducir las inequidades de desarrollo para la infancia (Eming y Fujimoto, 2004).

Aunque la interacción madre-niño y la estimulación en el hogar durante la infancia son insuperables, la atención y cuidados que los niños reciben en el CDI son cruciales. Esto lo evidencian trabajos como el de Peisner-Feinberg, Burchinal, Clifford, Culkin, Howes, Kagan et al. (2001) quienes encontraron un modesto, pero duradero efecto del cuidado recibido en el CDI en el desarrollo del lenguaje y rendimiento académico del niño durante su educación preescolar e inclusive hasta el segundo año de educación primaria.

Igualmente, en el trabajo de Vernon-Feagans, BratschHines, \& The Family Life Project Key Investigators (2013) se evidencia la asociación entre la calidez de la relación maestra-niño con las habilidades cognitivas y sociales de los años escolares siguientes. Incluso se ha sugerido que un cuidado de calidad en CDI aminora los efectos negativos de un bajo nivel de lenguaje en el hogar característico de familias en desventaja socioeconómica.

\section{Prácticas de cuidado y calidad de la interacción cuidadora-niño}

Los estudios que evalúan las condiciones y calidad del funcionamiento de los CDI's incluyen variedad de aspectos a ser evaluados (Araujo y López Boo, 2015; Dunn, 1993; 
Torres, 2014); la competencia profesional del personal que atiende directamente a los niños es uno de ellos. En su estudio, Arnett (1989), encuentra que el nivel de educación y entrenamiento de las cuidadoras se asocia positivamente con sus actitudes durante la interacción con los niños. Las cuidadoras con mayores niveles de entrenamiento fueron menos autoritarias y tuvieron puntajes más altos en interacciones positivas y más bajos en prácticas castigantes y de desapego con los niños.

En forma similar, Cassidy, Buell, Pugh-Hoese, \& Russell (1995), encontraron que los estudiantes que cursaban más créditos durante su formación profesional, punteaban mejor en manejo del grupo así como en tener expectativas del niño más acordes con su nivel de desarrollo. Lo anterior es consistente con los hallazgos reportados periódicamente por el National Institute of Children Health \& Human Development, uno de los proyectos de investigación sobre desarrollo infantil más reconocidos a nivel mundial (NICHD, 2015).

En educación inicial, las rutinas constituyen situaciones de aprendizaje que los niños realizan todos los días de forma estable y permanente y que fomentan la autonomía al exponerlos consistentemente a la norma social y al modelo de conducta esperado (Garbarino y Blasi, 2013). Las rutinas más importantes en este período son las de cuidado de la higiene, alimentación y sueño, las cuales a la vez que lo socializan protegen la salud y el bienestar del niño.

Por su importancia, rutinas como la limpieza de genitales, el lavado de manos y cara, entre otros, se encuentra definido dentro de las competencias profesionales de las cuidadoras en CDI's (SEP, 2007) de cuyo seguimiento se esperaría un menor riesgo de contagio en infecciones respiratorias y por parasitosis infantil que siguen siendo padecimientos comunes en CDI's, sobre todo entre los menores de un año (Alonzo Salomón y Roque Rodríguez, 2001; Nandí Lozano, Espinosa, Viñas Flores y Avila Figueroa, 2002; Giraldo Gómez, Lora, Henao, Mejía y Gómez Marín, 2005).

Por otra parte, la calidad de la relación afectiva entre las cuidadoras y los niños es un elemento crucial en el desarrollo socioafectivo y emocional de los pequeños, como lo han mostrado incontables estudios (Papalia, Olds y Feldman, 2005). En la relación con las madres o cuidadoras primarias se establece el apego del niño, que es un mecanismo de adaptación vital de donde emergerá su habilidad para relacionarse con sus iguales y con el resto de adultos (Ainsworth, Bell y Stayton, 1971).

De una relación de apego segura donde el niño sabe que su cuidadora está allí para protegerlo, se incentiva la conducta exploratoria del niño, fundamental para el desarrollo cognitivo (Garrido Rojas, 2006; Lafuente, 2000). De un trato afectuoso hacia el niño que fomenta la expresión de sus emociones y que recibe adecuada contención y enseñanza para manejar el estrés natural de la vida, surge la autorregulación emocional, precursora de la adaptación social del niño, basada en la confianza en los adultos que lo rodean, misma que sienta las bases de su personalidad (Papalia et al. 2005).
La norma técnica demanda de las cuidadoras una actitud afable, cálida y respetuosa por el peso que esto tiene para el desarrollo socioemocional del niño. En el caso de los lactantes, a quienes erróneamente se les puede atribuir inhabilidad para interactuar socialmente, la hora del biberón y cambio de pañal son los momentos para establecer esa comunicación con el niño a través de un intercambio en verbalizaciones, sonrisas y contacto físico (Aranda, 2000; Sumner y Spietz, 1994).

Adicionalmente se ha sugerido que para las cuidadoras en CDI's es importante que tengan un conocimiento básico sobre desarrollo infantil dado que este impacta su habilidad para el desarrollo de las actividades programáticas, así como sus expectativas respecto de lo que cada niño debe lograr. Guerra, Campaña, Fredes, Gutierrez y Plaza (2011), encontraron que el cambio en creencias irracionales sobre la crianza (p. ej., la idea de que nada de lo que haga el cuidador hará cambiar la conducta de un niño dificil) condujo tanto a las madres como a las cuidadoras a una mayor capacidad para el manejo conductual de los niños. En su estudio se señala que el entrenamiento en desarrollo infantil y prácticas asociadas al manejo conductual, redujo la frecuencia en conductas disruptivas del niño tanto en el hogar como en el CDI.

El hecho de que el $76 \%$ de los niños que asisten a un CDI permanecen en él desde cuatro hasta ocho horas diarias (INEGI, 2014), habla de la importancia que tiene analizar la calidad de las prácticas e interacción cotidiana entre las cuidadoras y los niños. Por lo anterior, el objetivo de este estudio es describir dichas prácticas en un grupo de cuidadoras de CDI's de la región; particularmente, las prácticas referidas a cuidados de salud e higiene, manejo conductual y de emergencias, conocimiento sobre desarrollo infantil, y calidad de la interacción con el niño. A partir de esta descripción, se pretende discutir las implicaciones que la calidad de estas prácticas tiene sobre el desarrollo de los niños.

\section{Método}

\section{Participantes}

El estudio se realizó con una muestra a conveniencia de 55 cuidadoras de CDI quienes accedieron de manera libre y voluntaria a participar. En promedio, las participantes tenían 27 años de edad, una experiencia como cuidadoras de 4.75 años, y su nivel educativo fluctuaba de preparatoria terminada hasta algunos años de licenciatura. Fueron empleadas 22 cuidadoras de CDI privados, 18 de CDI públicos (IMSS, SEDESOL, DIF) nueve de mixtos y seis no respondieron.

\section{Instrumento}

Se elaboró exprofeso un cuestionario de 84 reactivos basados en instrumentos existentes que se integraron de la siguiente manera, y que las cuidadoras contestaron usando en su mayoría escalamiento tipo likert.

\section{Prácticas de cuidado}

De la Norma Técnica de Competencia Laboral NCASS007.01 (SEP, 2007) se tomaron cinco reactivos para

\section{Savia}


evaluar las prácticas de cuidado a la salud e higiene, que serían contestados en una likert de cinco puntos [5 (siempre) a 1 (nunca)], ejemplo de un reactivo es: ¿Al cambiar de pañal o en la bacinica limpia los genitales del niño de enfrente hacia atrás? Para el apartado de prácticas de cuidado en el manejo de las contingencias, la norma técnica no es específica; por esta razón, se elaboraron ocho reactivos que indagaban sobre la percepción de las cuidadoras en su capacidad para ejecutar acciones como: manejar un niño que se ahoga con algún alimento o juguete, darle primeros auxilios (respiración boca a boca) a un niño, entre otros.

Estos reactivos debían contestarse usando una likert de cuatro puntos [3 (totalmente) a 0 (nada)]. Igualmente, la norma implícitamente señalaba la competencia en el manejo conductual de los niños, pero no especificaba situaciones, por lo que se elaboraron cinco reactivos con el mismo escalamiento de cuatro puntos que indagaban cosas como: ¿quétan preparada te sientes para manejar el berrinche de un niño? ¿...para manejar a un niño que muerde a los demás?, entre otros. En todos los casos, la direccionalidad de los reactivos indicaba que a mayores puntajes mayor competencia.

El alpha de Cronbach para estos conjuntos de reactivos fueron de $@=.68$ para cuidado a la salud e higiene, $@=.79$ para cuidado en el manejo de las emergencias, y $@=.72$ para cuidado en el manejo conductual de los niños. Por último, se incluyeron dos reactivos sobre entrenamiento en control de esfínteres, también requerido en la norma de competencia; el primero era abierto y preguntaba: ¿a qué edad inicia usted con el control de esfinteres? y el segundo indagaba: ¿qué tiempo aproximado permanecen los niños sentados en las bacinicas? Estos incluyeron tres opciones de respuesta (3 minutos, 10 minutos, 30 minutos, con base en lo que la norma estipulaba como el tiempo máximo para esta práctica).

\section{Calidad de la interacción cuidador-niño}

Se seleccionaron del NCAST caregiver/parent-child interaction feeding/teaching manual (SumNer \& Spietz, 1994) 14 reactivos para conocer la calidad de la interacción entre la cuidadora y el niño durante rutinas de alimentación o actividades programáticas con los niños. El NCAST define la calidad de la interacción a partir de la sensibilidad, responsividad y contingencia en la respuesta del cuidador a las señales del niño. Se les pidió a las cuidadoras que reportaran con un escalamiento de cinco puntos [5 (siempre) a 1 (nunca)], la frecuencia con la cual ellas se comportaban de maneras señaladas.

Ejemplos de reactivos para esta sección son: ¿con qué frecuencia le sonríe, verbaliza, y/o elogia al niño durante la interacción?, ¿con qué frecuencia logra la atención del niño antes de iniciar con una interacción? El alpha de Cronbach para estos conjuntos de reactivos fue de $@=.92$ para las maestras del área de lactantes (7 cuidadoras), y $@=.69$ para las maestras de maternal (48 cuidadoras).

\section{Conocimiento sobre desarrollo infantil}

Se seleccionaron 33 de los 85 reactivos incluidos en el Developmental Checklist. Birth to Five (Shelov, \& Hannemann, 1994) para evaluar el nivel de conocimiento sobre desarrollo infantil de las cuidadoras. Los 33 reactivos incluidos en el cuestionario cubrían logros de desarrollo de niños en todos los dominios: físico/psicomotriz, cognitivo, lenguaje, afectivo y social. Ejemplo de reactivos son: ¿a qué edad en promedio el niño ya es capaz de seguir visualmente objetos en movimiento?, y ¿a qué edad es capaz de meter y sacar objetos de un contenedor (cubos, dados, etc.)?, entre otros.

Las cuidadoras debieron responder a cada reactivo señalando en qué rango de edad se espera que el niño logre la acción, con seis opciones de respuesta ( 0 a 3 meses, 4 a 7 meses, 8 a 12 meses, 12 a 24 meses, 25 a 36 meses, y 4 a 5 años). Las respuestas se codificaron como correcta o incorrecta, y se generó un promedio de aciertos para cada participante.

\section{Procedimiento}

Con el apoyo de un grupo de estudiantes universitarios de la carrera de psicología, previamente entrenados por su maestra, se invitó personalmente a las cuidadoras a contestar la entrevista de manera libre y voluntaria. Las entrevistas se llevaron a cabo en su mayoría en los centros de trabajo de las cuidadoras durante su tiempo de descanso o a la salida de sus labores. La entrevista duró aproximadamente 45 minutos.

\section{Resultados}

Se hicieron análisis de frecuencia a nivel reactivo para conocer específicamente qué prácticas y episodios de interacción son más comunes entre las cuidadoras y los niños. Se estimó la media aritmética de ejecución diaria por tipo de práctica (salud e higiene, manejo conductual, manejo de emergencias y entrenamiento de esfínteres), de episodios de la interacción y de conocimientos sobre desarrollo infantil. Por último, se ejecutó un análisis de correlación bivariada con todas las escalas analizadas para estimar las posibles asociaciones entre prácticas, interacción y conocimientos. 
Como se observa en la tabla 1, las cuidadoras reportan alta frecuencia en prácticas como: la limpieza de genitales y cambio de ropa prenda por prenda con $78.4 \%$ y 81.4 $\%$ de cuidadoras que lo hacen siempre o frecuentemente, respectivamente. Sin embargo, las cuidadoras reportan que usan el peine personal del niño y el monitoreo de su temperatura con menor frecuencia dado el $33.3 \%$ y 27.5 $\%$, respectivamente, que reportan hacerlo desde a veces hasta nunca. El promedio de ejecución diaria (cuidadoras que lo hacen siempre) para el conjunto de las cinco prácticas de salud e higiene es de $54.9 \%$.

\section{Tabla 1}

\begin{tabular}{|c|c|c|}
\hline \multicolumn{2}{|l|}{ Reactivo } & \multirow{2}{*}{$\frac{\%}{70.6}$} \\
\hline Cambia el pañal y/o limpia los & siempre & \\
\hline genitales del niño de enfrente & frecuentemente & 7.8 \\
\hline \multirow[t]{3}{*}{ hacia atrás } & a veces & 7.8 \\
\hline & casi nunca & 3.9 \\
\hline & nunca & 9.8 \\
\hline Cambia de ropa al niño prenda & siempre & 55.9 \\
\hline \multirow[t]{4}{*}{ por prenda } & frecuentemente & 25.5 \\
\hline & a veces & 19.6 \\
\hline & casi nunca & 2.0 \\
\hline & nunca & 0 \\
\hline Coloca a los lactantes de cubito & siempre & 45.8 \\
\hline \multirow[t]{4}{*}{ lateral a la hora de dormir } & frecuentemente & 22.9 \\
\hline & a veces & 8.3 \\
\hline & casi nunca & 2.1 \\
\hline & nunca & 20.8 \\
\hline Los peina con su peine personal & siempre & 49.1 \\
\hline \multirow[t]{4}{*}{ respetando su peinado } & frecuentemente & 19.6 \\
\hline & a veces & 23.5 \\
\hline & casi nunca & 3.9 \\
\hline & nunca & 5.9 \\
\hline Cuando están dormidos, verifica la & siempre & 52.9 \\
\hline frecuencia de la respiración y la & frecuentemente & 19.6 \\
\hline \multirow[t]{3}{*}{ temperatura } & a veces & 15.7 \\
\hline & casi nunca & 2.0 \\
\hline & nunca & 9.8 \\
\hline
\end{tabular}

Porcentaje de reporte de prácticas de atención a la salud e higiene, $\mathrm{N}=55$ Nota: la tabla muestra los porcentajes de una muestra que varía su tamaño con un promedio de valores perdidos de $1 \%$ a nivel reactivo.

Para el control de esfínteres (no incluido en tabla 1) el $71 \%$ de las cuidadoras lo inician dentro del rango de edad adecuada (18 a 24 meses), con el $100 \%$ de ellas cumpliendo con el criterio de permanencia en la bacinica (10 minutos como máximo), tal como se prescribe en la norma técnica de competencia.

Respecto a la capacidad para el manejo conductual y de contingencias (ver tabla 2), se observa, en general, una percepción de competencia de moderada a buena, según se evidencia en el porcentaje de cuidadoras que reporta sentirse capaz regular o totalmente de manejar las distintas situaciones. En particular, las referidas al manejo conductual, casi el $100 \%$ de las cuidadoras reportan sentirse capaces en la mayoría de las situaciones (berrinche, pelea entre niños, etc.), con excepción del manejo de un niño retraído donde un $7.3 \%$ se percibe casi nada capaz de manejarlo.

Sin embargo, en el manejo de las contingencias, las cuidadoras se perciben un poco menos preparadas. Esto se evidencia con el $30.9 \%$ de cuidadoras que reportan sentirse casi nada capaces de manejar a un niño que convulsiona, o el $21.8 \%$ que se perciben pobremente capaces de dar primeros auxilios (respiración de boca a boca a un niño). En promedio, los cinco reactivos de manejo conductual tienen el $66.9 \%$ de cuidadoras que reportan sentirse totalmente capaces de manejar las situaciones; mientras que para el manejo de contingencias el promedio es de $51.4 \%$.

La tabla 3 describe las frecuencias de los episodios de calidad en la interacción cuidadora-niño. Como se puede observar, en diez de los 14 reactivos, el $80 \%$ aproximadamente de las cuidadoras reporta ejecuciones de siempre a frecuentemente, lo cual habla de un nivel moderadamente alto de interacciones de calidad. Aun así, el promedio de interacciones que se ejecutan siempre por las cuidadoras alcanza solo el $45 \%$, lo cual indica que pese al buen nivel de frecuencia general, algunas cuidadoras reportan una frecuencia menor de interacciones de calidad.

Específicamente, en los reactivos: permitirle al niño explorar los objetos antes de iniciar la instrucción, detener la actividad ante señales de malestar del niño, distraerlo orientando su atención a un nuevo objeto, y no utilizar menos de un minuto ni más de cinco para enseñarle al niño la ejecución que se le solicita, al menos un $20 \%$ de las cuidadoras reportan una frecuencia que va de a veces a nunca. Lo anterior es un dato revelador por las posibles carencias estimulantes para el desarrollo del niño dadas por la falta de habilidad de la cuidadora para propiciarlas.

Respecto al nivel de conocimiento sobre desarrollo infantil (no se muestra en las tablas), el promedio general del grupo de cuidadoras fue de $39.8 \%$ de respuestas correctas para el conjunto de 33 reactivos. Aun cuando el promedio es bajo, en algunas áreas del desarrollo, las cuidadoras evidencian un mayor conocimiento. Tal es el caso de aspectos del desarrollo motriz y de lenguaje donde reactivos como: el niño se pasa objetos de una mano a otra (81.1\% correctas), el niño se viste o se desviste solo (76.9\%), el niño camina solo (67.9\%) y el niño platica historias largas (61.5\%) alcanzaron las frecuencias más altas de respuestas correctas. Mientras que el desarrollo socioafectivo parece ser el área donde las cuidadoras tienen menor nivel de conocimiento, con reactivos como: el niño empieza a desarrollar la sonrisa social (94.3\%), el niño se interesa por su imagen en el espejo (82.7 \%) y empieza el juego de roles, tuvieron las frecuencias más altas de respuesta incorrecta (86.8\%).

\section{Savía}


Tabla 2

Percepción de competencia en el manejo conductual y de contingencias, $\mathrm{N}=55$

Reactivo

Sabe manejar el berrinche de un

totalmente

casi nada

Sabe manejar a un niño que muerde nada

talmente $\quad 65.5$

regular

casi nada

Sabe manejar a un niño que se

ahoga con algún alimento o

juguete

Sabe manejar a un niño que se

cae/lesiona con posible fractura

Sabe manejar a un niño que se

desmaya o convulsiona

En caso de incendio o sismo es

capaz de mantener la calma

Es capaz de seguir los pasos

recibidos en entrenamiento para

casos de emergencias

Sabe controlar a un grupo de niños asustados

totalmente

regular

casi nada

nada

totalmente

regular

casi nada

nada

totalmente

regular

casi nada

nada

totalmente

regular

casi nada

nada

totalmente

regular

casi nada

nada

totalmente

regular

casi nada

nada

totalmente

regular

casi nada

nada

Sabe realizar acciones como evacuar

niños, moverse en un lugar con

humo, manejar los extinguidores

Sabe darle primeros auxilios a un

niño (respiración de boca a boca)

$\begin{array}{cc}\text { nada } & 0 \\ \text { totalmente } & 63.6 \\ \text { regular } & 32.7 \\ \text { casi nada } & 3.6 \\ \text { nada } & 0 \\ \text { totalmente } & 34.5 \\ \text { regular } & 43.6 \\ \text { casi nada } & 20.0 \\ \text { nada } & 1.8\end{array}$

Nota: la tabla muestra los porcentajes de una muestra que varía su tamaño con un promedio de valores perdidos de $1 \%$ a nivel reactivo. 
Tabla 3

Porcentaje en reporte de interacción cuidadora-niño/a, N = 48

\begin{tabular}{|c|c|c|}
\hline Reactivo & & $\%$ \\
\hline Logra la atención del niño antes & siempre & 31.3 \\
\hline \multirow[t]{4}{*}{ de iniciar con una instrucción } & frecuentemente & 60.4 \\
\hline & a veces & 8.3 \\
\hline & casi nunca & 0 \\
\hline & nunca & 0 \\
\hline Le permite al niño que & siempre & 39.6 \\
\hline explore los objetos/materiales & frecuentemente & 37.5 \\
\hline unos minutos antes de iniciar & a veces & 18.8 \\
\hline \multirow[t]{2}{*}{ la instrucción } & casi nunca & 2.1 \\
\hline & nunca & 2.1 \\
\hline Le sonríe, verbaliza y/o elogia & siempre & 85.4 \\
\hline al niño durante la & frecuentemente & 12.5 \\
\hline \multirow[t]{3}{*}{ interacción } & a veces & 2.1 \\
\hline & casi nunca & 0 \\
\hline & nunca & 0 \\
\hline Varía la posición del niño o de & siempre & 42.6 \\
\hline los objetos y materiales & frecuentemente & 44.7 \\
\hline después de varios intentos & a veces & 10.6 \\
\hline \multirow[t]{2}{*}{ fallidos del niño } & casi nunca & 0 \\
\hline & nunca & 2.1 \\
\hline Detiene la actividad ante & siempre & 22.9 \\
\hline señales de malestar del niño & frecuentemente & 31.3 \\
\hline \multirow[t]{3}{*}{ (falta de atención, enojo) } & a veces & 31.3 \\
\hline & casi nunca & 12.5 \\
\hline & nunca & 2.1 \\
\hline Distrae al niño orientando su & siempre & 21.3 \\
\hline \multirow[t]{4}{*}{ atención a un nuevo objeto } & frecuentemente & 29.8 \\
\hline & a veces & 25.5 \\
\hline & casi nunca & 21.3 \\
\hline & nunca & 2.1 \\
\hline Evita movimientos abruptos y & siempre & 75.0 \\
\hline comentarios negativos al & frecuentemente & 14.6 \\
\hline \multirow[t]{3}{*}{ niño } & a veces & 2.1 \\
\hline & casi nunca & 2.1 \\
\hline & nunca & 6.3 \\
\hline Evita hablar por encima de las & siempre & 54.3 \\
\hline \multirow[t]{4}{*}{ vocalizaciones del niño } & frecuentemente & 32.6 \\
\hline & a veces & 4.3 \\
\hline & casi nunca & 4.3 \\
\hline & nunca & 4.3 \\
\hline Arregla el escenario para la & siempre & 45.8 \\
\hline instrucción (p. ej. libre de & frecuentemente & 45.8 \\
\hline \multirow[t]{3}{*}{ distracciones) } & a veces & 8.3 \\
\hline & casi nunca & 0 \\
\hline & nunca & 0 \\
\hline
\end{tabular}




$$
\begin{gathered}
\text { Utiliza al menos dos frases } \\
\text { distintas para describir la } \\
\text { ejecución del niño }
\end{gathered}
$$

Utiliza instrucción verbal y

moldeamiento de la ejecución

del niño

Le da palmaditas,

abrazos, besos al niño

durante la interacción

Elogia el logro parcial de la

ejecución del niño y no solicita

más de un par de veces la

ejecución ya lograda

por el niño

Utiliza no menos de 1 minuto

ni más de 5 minutos

enseñándole la ejecución

al niño siempre

frecuentemente

52.1

a veces

12.5

casi nunca

nunca

2.1

siempre

51.1

frecuentemente $\quad 44.7$

a veces

4.3

casi nunca

0

nunca

0

siempre

56.3

frecuentemente $\quad 31.3$

a veces $\quad 12.5$

casi nunca 0

nunca 0

siempre $\quad 45.7$

frecuentemente $\quad 34.8$

a veces $\quad 17.4$

casinunca $\quad 2.2$

nunca 0

siempre $\quad 27.7$

frecuentemente $\quad 36.2$

a veces 31.9

casi nunca 2.1

nunca

Nota: se incluyeron únicamente las cuidadoras que atienden niños(as) en grupo de maternal por ser la mayoría de las participantes.

Respecto al nivel de conocimiento sobre desarrollo infantil (no se muestra en las tablas), el promedio general del grupo de cuidadoras fue de $39.8 \%$ de respuestas correctas para el conjunto de 33 reactivos. Aun cuando el promedio es bajo, en algunas áreas del desarrollo, las cuidadoras evidencian un mayor conocimiento. Tal es el caso de aspectos del desarrollo motriz y de lenguaje donde reactivos como: el niño se pasa objetos de una mano a otra ( $81.1 \%$ correctas), el niño se viste o se desviste solo (76.9\%), el niño camina solo (67.9\%) y el niño platica historias largas (61.5\%) alcanzaron las frecuencias más altas de respuestas correctas. Mientras que el desarrollo socioafectivo parece ser el área donde las cuidadoras tienen menor nivel de conocimiento, con reactivos como: el niño empieza a desarrollar la sonrisa social (94.3\%), el niño se interesa por su imagen en el espejo (82.7 \%) y empieza el juego de roles, tuvieron las frecuencias más altas de respuesta incorrecta (86.8 \%).

Por último, se computó un análisis de correlación bivariado entre las características de las cuidadoras (edad, experiencia como cuidadoras y nivel de estudios), con las prácticas, las interacciones y el conocimiento para explorar si existe asociación entre ellas que oriente en la profundización de su estudio. La tabla 4 muestra correlaciones significativas entre la edad de la cuidadora y la capacidad percibida para manejar conductualmente a los niños $\left(.285^{*}\right)$, así como con su nivel de conocimientos sobre desarrollo infantil $(.279 *)$ indicando que a mayor edad más capacidad y conocimiento.

\begin{tabular}{|c|c|c|c|c|c|c|c|c|}
\hline Variable & 1 & 2 & 3 & 4 & 5 & 6 & 7 & 8 \\
\hline Edad & 1 & $.632^{* *}$ & .250 & .134 & $.285^{*}$ & .210 & .154 & $.279^{*}$ \\
\hline Experiencia & & 1 & $.289^{*}$ & .074 & .166 & .060 & .034 & .117 \\
\hline Nivel de estudios & & & 1 & -.171 & $.343^{*}$ & .212 & .143 & .206 \\
\hline Prácticas de salud e higiene & & & & 1 & .086 & .167 & $.288^{*}$ & -.191 \\
\hline Manejo conductual & & & & & 1 & $.431^{*}$ & .074 & -.060 \\
\hline Manejo de contingencias & & & & & & 1 & $.295^{*}$ & .121 \\
\hline Calidad de la interacción & & & & & & & 1 & -.060 \\
\hline Nivel de conocimientos & & & & & & & & 1 \\
\hline
\end{tabular}

Tabla 4

Correlación bivariada entre características de las cuidadoras y prácticas de cuidado, interacción y conocimientos

Nota: ${ }^{*} p<.05,{ }^{* *} p<.01$ 
Igualmente, observamos que el nivel de estudios se asocia positivamente con el manejo conductual $\left(.343^{*}\right)$, que las prácticas de salud e higiene se correlacionan positivamente con los episodios de calidad en la interacción $\left(.288^{*}\right)$ y con el manejo de contingencias $\left(.295^{*}\right)$ ambas en el sentido esperado.

\section{Discusión y conclusiones}

Según la Secretaría de Educación Pública, a partir del 2000 todo personal que labora en los CDI's debe tener un perfil psicolaboral donde su nivel de educación oficial, sus conocimientos, actitudes y habilidades sean propias para fomentar el desarrollo infantil (SEP, 2007). Para el caso de nuestras cuidadoras, en general, se observa un nivel de moderado a bajo en sus prácticas de cuidado y calidad de su interacción.

En particular, el entrenamiento de esfinteres parece ser una de las prácticas más correctamente ejecutadas, la inmensa mayoría de las cuidadoras lo establece dentro del rango de edad correcto, y la totalidad de ellas no excede el tiempo de bacinica según lo exige la norma. Esto es una buena práctica que respeta la madurez del niño, evita forzar una tarea, y fomenta la individualidad y autonomía del niño, según lo plantean la mayoría de las teorías del desarrollo psicoafectivo para esta etapa (Papalia et al. 2005).

Por otro lado, aunque las prácticas de salud e higiene son realizadas con una frecuencia deseable, estas son tareas que requieren de una ejecución ejemplar por las implicaciones que tienen sobre la salud del niño. Tan solo una vez que se realice inadecuadamente la limpieza del área del pañal, es suficiente para poner al niño en riesgo de una infección por contacto con heces fecales.

El uso indistinto del peine puede transmitir parásitos de un niño a otro, y el olvido ocasional en lavado de manos y cara puede provocar contagio de infecciones respiratorias y estomacales por el intercambio constante de objetos que los niños a esta edad normalmente llevan a sus bocas (Alonzo Salomón y Roque Rodríguez, 2001; Nandí Lozano et al., 2002; Giraldo Gómez et al. 2005). Esto resulta de relevancia porque durante la primera infancia el sistema inmunológico del niño está aún en desarrollo y es más proclive a todo tipo de contagios (Araujo y López Boo, 2015).

Un aspecto favorable del grupo de cuidadoras es la percepción que tienen respecto de su capacidad para el manejo conductual de los niños, lo que es de suma importancia porque el control del grupo de niños fomenta un ambiente ordenado necesario para el aprendizaje, con reglas de conducta y modelos de ejecución (Hamre y Pianta, 2001; Mashburn et al. 2008). El manejo conductual del grupo, acompañado de la atención sensible y afectuosa de las necesidades particulares de cada niño, implican habilidad de la cuidadora para mediar en los conflictos, y para estimular la autorregulación y expresión emocional del niño.

Esta cuidadora hábil se convierte así en la base segura con quien el niño establece una relación de seguridad y confianza en el centro, semejante a la relación que se tiene con una madre igualmente hábil en el hogar (Ainswort et

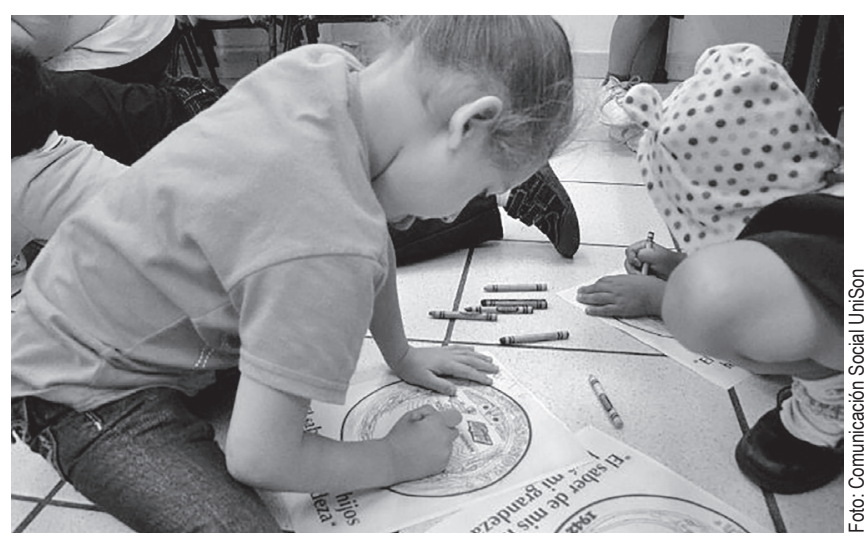

al. 1971). Este lazo de apego del niño con sus cuidadoras primarias es, a su vez, imprescindible para su desarrollo cognitivo (Garrido Rojas, 2006).

Sin embargo, se reporta una baja frecuencia de episodios interactivos de calidad que están directamente vinculados con el desarrollo cognitivo del niño, como es el no permitirle que explore con los objetos con los que se espera realice una actividad programática, o el no dedicar el tiempo adecuado para explicarle la tarea al niño. Estas situaciones reflejan el desconocimiento de las cuidadoras del rol fundamental de la exploración y de la explicación en el proceso de aprender de los niños en edades lactantes y maternales (Sumner y Spitz, 1994).

Igualmente, nuestros datos arrojan información que se convierte en focos rojos para el bienestar y seguridad de los niños en los centros. Nuestras entrevistadas reportaron sentirse menos capaces de manejar algunas situaciones comunes que implican emergencia, por ejemplo, atender a un niño que se ahoga con un juguete o manejar a un niño que se ha caído con posibilidad de fractura. Esto merecería una atención mayor por parte de las autoridades para entrenar al personal de los centros y evitar las consecuencias graves de accidentes comunes en ambientes infantiles.

Un dato de interés sin duda es la no asociación entre el nivel de estudios de la cuidadora y su nivel de conocimientos sobre desarrollo infantil. Al parecer, nuestras cuidadoras más preparadas son las que mayor edad tienen (.279*) sugiriéndose que es la propia experiencia en las labores lo que las dota de conocimientos sobre el tema y las capacita en el manejo conductual de los niños, y no tanto la educación formal en el campo de estudio.

Por otro lado, un buen nivel de conocimiento sobre desarrollo infantil es pieza clave para la detección temprana de un retraso en el desarrollo y por ende en su recuperación (Craig y Baucum, 2009). La formación profesional en detección de necesidades educativas especiales es una obligación de los centros de educación inicial, misma que aún no ha sido satisfecha a cabalidad ni en la región, ni a nivel nacional (Valencia, 2014).

Aun cuando los logros en motricidad y del lenguaje fueron de las áreas más fácilmente identificables por las cuidadoras y las que han recibido más atención en cuanto a educación especial, el desarrollo socioafectivo es igualmente importante y su retraso acarrea consecuencias en la

\section{Savia}


capacidad del niño de relacionarse con los otros niños y adultos, limitando así su ajuste al ambiente escolar (Hamre y Pianta, 2001; Garrido Rojas, 2006). La elevada frecuencia de respuesta errónea para identificar, por ejemplo, la aparición de la sonrisa social puede fomentar la indiferencia de una cuidadora que desconoce el significado de la misma.

La sonrisa social es el momento idóneo para la interacción entre la cuidadora y el niño; si esta interacción es contingente, empática y responsiva a las señales del niño, se facilita la comunicación y el afecto que fomentan el lazo de apego entre el niño y la cuidadora, y que a su vez influyen en las otras áreas del desarrollo del niño (Papalia et al. 2005).

Existe un vacío en información sistematizada de cómo se desarrollan las acciones cotidianas entre cuidadoras y niños en los centros. Idealmente, estas profesionales poseen adecuada formación en conocimiento y habilidades para su trabajo, así como un trato cálido cotidiano que provea al niño de un ambiente seguro, protegido y feliz. Con base en nuestros datos esto no se cumple al cien por ciento.

El nivel de competencia ofrece áreas de oportunidad que los responsables de los centros deberían atender y las autoridades deberían monitorear, regular y evaluar; pues de la calidad de estas prácticas cotidianas depende en buena parte la salud y el bienestar de los niños bajo su responsabilidad.

El estudio adolece de importantes limitaciones; un muestreo por conveniencia y un tamaño reducido de muestra no permiten generalizaciones; por tanto, la calidad en prácticas e interacciones de nuestras cuidadoras no se pueden considerar representativas del resto de ellas. Otra limitación es la deseabilidad social cuando se utiliza el autorreporte. En futuros trabajos seria muy conveniente la utilización de medidas convergentes como registros observacionales, especialmente para las prácticas e interacciones cuidadoraniño con miras a reducir esa fuente de error.

Pese a la naturaleza exploratoria y alcance descriptivo de este estudio que surge como un ejercicio de contraloría social, creemos que los hallazgos aquí reportados son relevantes por su novedad en países como México, donde las políticas orientadas al fomento de la infancia, del respeto a sus derechos y a la búsqueda de su bienestar, todavía carecen de programas y evaluaciones que indiquen el rumbo a seguir.

\section{Referencias}

Ainsworth, M.D.S., Bell, S.M., \& Stayton, D.J. (1971). Attachment, exploration, and separation: Illustrated by the behavior of one-year-olds in a strange situation. Child Development, 41, 49-67.

Alonzo Salomón, J. y Roque Rodríguez, O. (2001). Incidencia de diarrea infantil en una guardería. Revista Biomédica, 12, 256-261.

Aranda, R. (2000). La autoestima y las habilidades sociales en edades tempranas. Congreso mundial de lectoescritura, celebrado en Valencia. Universidad Autónoma de Madrid. Recuperado el 12 de agosto 2013 de www.waece.org/biblioteca/pdfs/d134.pdf
Araujo, M.C. y Lopez Boo, F. (2015). Los servicios de cuidado infantil en América Latina y el Caribe. El trimestre económico, LXXXII (2), 326, 249-275.

Arnett, J. (1989). Caregivers in day-care centers: Does training matter? Journal of Applied Developmental Psychology, 10(4), 541-552.

Carrion, V.G. (2010). Can traumatic stress damage the brain? Understanding the effect of early trauma on brain development. En C. Polk (presidencia). Conferencia magistral llevada a cabo en el 25th National Training Institute (NTI): Connecting science, policy, and practice, Phoenix, Arizona.

Cassidy, D.I., Buell, M.I., Pugh-Hoese, S., \& Russell, S. (1995). The effect of education on child care teachers' beliefs and classroom quality: Year one evaluation of the TEACH early childhood associate degree scholarship program. Early Childhood Research Quarterly, 10(2), 171183.

Craig, G.J. y Baucum, D. (2009). Desarrollo Psicológico. México: Pearson Prentice Hall.

Dunn, L. (1993). Proximal and distal features of day care quality and children's development. Early Childhood Research Quarterly, 8(2), 167-192.

Eming, Y.M. y Fujimoto, G.G. (2004). Desarrollo infantil temprano: lecciones de los programas no oficiales. Acta Pedagógica, 13(2), 186-198.

Garbarino, P. y Blasi, C. (2013). Recorridos didácticos en la educación inicial. Qué es un CENDI. Recuperado de www.sev.gob.mx/subdeseduc/diep...2013/1.../ QueEsUnCENDI.Pdf

Garrido Rojas, L. (2006). Apego, emoción y regulamiento emocional. Implicaciones para la Salud. Revista Latinoamericana de Psicologia, 38(3), 493-507

Giraldo Gómez, J.M., Lora, F., Henao, L.H., Mejía, S. y Gómez Marín, J.E. (2005). Prevalencia de giardiasis y parásitos intestinales en preescolares de hogares atendidos en un programa estatal en Armenia, Colombia. Revista de Salud Pública, 7(3), 327-338

Gómez, E., Muñoz, M.M. y Haz, A.M. (2007). Familias multiproblemáticas y en riesgo social: características e intervención. Psykhe, 16(2), 43-54

Grusec, J.E. (2002). Parental socialization and children's acquisition of values. In M.H. Bornstein (ed.). Handbook of Parenting, vol. 5, (pp. 143-168). Mahwah, NJ: Lawrence Erlbaum.

Guerra, C., Campaña, M., Fredes, V., Gutierrez, L. y Plaza, H. (2011). Regulación de la agresividad entre preescolares mediante el entrenamiento a madres y profesoras. Terapia Psicológica, 29(2), 197-211.

Hamre, B.K., \& Pianta, R.C. (2001). Early teacher-child relationships and the trajectory of children's school outcomes through eighth grade. Child development, 72(2), 625-638. 
Harper, C., Alder, H., \& Pereznieto, P. (2012). Escaping poverty traps. Children and Chronic poverty. In I. Ortiz, L. Moreira, \& S. Engilbertsdóttir (eds.). Child poverty and inequality. New Perspectives. New York: UNICEF Division of Policy and Practice. Recuperado de http:// www.unicef.org/socialpolicy/files/Child_Poverty_ Inequality_FINAL_Web_web.pdf

Instituto Mexicano del Seguro Social (IMSS). (2012). Encuesta Nacional de Satisfacción a Usuarios de Guarderías. Recuperado de http://www.imss.gob. $\mathrm{mx} /$ servicios/encuesta/Documents/2012Sep_ GuardResultados.pdf

Instituto Nacional de Estadística y Geografía (INEGI). (2014). Encuesta Nacional de Empleo y Seguridad Social 2013 (ENESS). Principales Resultados 2014. Recuperado de http://internet.contenidos.inegi.org. $\mathrm{mx} /$ contenidos/productos//prod_serv/contenidos/ espanol/bvinegi/productos/encuestas/hogares/ eness/2014/702825058777.pdf

Lafuente, M.J. (2000). Patrones de Apego, pautas de interacción familiar y funcionamiento cognitivo (de la década de los 70 a la década de los 90). Revista de Psicología General y Aplicada, 53(1), 165-190

López, F., Apodaca, P., Etxebarria, I., Fuentes, M.J. y Ortiz, M.J. (1998). Conducta prosocial en preescolares. Infancia y Aprendizaje, 82, 45-61.

Mashburn, A.J., Pianta, R.C., Hamre, B.K., Downer, J.T., Barbarin, O.A., Bryant, D., ... Howes, C. (2008). Measures of classroom quality in prekindergarten and children's development of academic, language, and social skills. Child development, 79(3), 732-749.

Movimiento 5 de Junio. (2011). Ley General de Prestación de Servicios para la Atención, Cuidado y Desarrollo Integral Infantil. Gobierno de la República. Diario Oficial de la Federación del 24 de octubre de 2011. Recuperado de http://www.movimiento5dejunio.org/abc/wp-content/ uploads/2012/05/PUBLICACION-DE-LEY-5-DEJUNIO-EN-DOF-24-OCT.2011.pdf

Myers, R. (1992). The twelve who survive. London: Routledge.

Nandí Lozano, E., Espinosa, L.E., Viñas Flores, L. y Avila Figueroa, C. (2002). Infección respiratoria aguda en niños que acuden a un centro de desarrollo infantil. Salud Pública de México, 44(3), 201-206.

National Institute of Children Health \& Human Development. NICHD. Recuperado de https://www. nichd.nih.gov/research/supported/seccyd/Pages/ overview.aspx

Nuñez, S. (2012). Mujeres, guarderías y exclusión, participación de las madres usuarias de guarderías del IMSS de frente a la nueva Ley de Estancias Infantiles (Tesis de maestría). Universidad de Sonora, Hermosillo, Sonora, México.

Papalia, D.E., Olds, S.W. y Feldman, R.D. (2005). Desarrollo Humano. México: McGraw Hill (trabajo original publicado en 1978).
Peisner-Feinberg, E.S., Burchinal, M.R., Clifford, R.M., Culkin, M.L., Howes, C., Kagan, S.L. y Yazejian, N. (2001). The relation of preschool child-care quality to children's cognitive and social developmental trajectories through second grade. Child development, 72(5), 15341553.

Sen, A. (1999). Romper el ciclo de la pobreza: Invertir en la infancia. París, 14 de marzo de 1999. Recuperado de http://www.eumed.net/cursecon/economistas/textos/ Sen\%20pobreza\%20e\%20infancia.htm\#_ftnref1

Secretaría de Educación Pública (SEP). (2007). Norma Técnica de Competencia Laboral NCASS007.01. CONOCER. Fecha de publicación: 14 de diciembre 2007. Recuperado de http://www.conocer.gob.mx/ seccionesExtras/reconocer/pof/EC0049.pdf

Shelov, S.P., \& Hannemann, R.E. (1994). Caring for your baby and young child birth to age 5. The complete and authoritative guide. Developmental Checklist. Birth to five. New York: Bantam Doubleday Dell Pub. Recuperado de http:// www.preschoollearningcenter.org/images/upload/ developmental_checklist.pd

Sotomayor Peterson, M. y Pineda León, M. (2014). Familia y Centro de Desarrollo Infantil: Una primera aproximación al diagnóstico de la situación en Sonora. En M. Montiel Carbajal, M. Domínguez Guedea, I.Y. Pérez Campos, M. Pineda León, M. Rivera Sander, M. Sotomayor Peterson y E. Valenzuela Medina, Intervenciones con enfoque psicosocial (pp. 25-48). Hermosillo, Sonora: Universidad de Sonora.

Sumner, G., \& Spietz, A. (Eds.). (1994). NCAST caregiver/ parent-child interaction teaching manual. Seattle: NCAST Publications, University of Washington, School of Nursing.

Tierney, A.L., \& Nelson, C. (2010). Brain development and the role of experience in the early years. Journal of Zero to Three: National Center for Infants, Toddlers, and Families, Special Issue December, 11-15.

Torres, A.L. (2014). Centros de cuidado y desarrollo infantil temprano en México: Análisis, desde una perspectiva de derechos, del Programa de Guarderías del IMSS y del Programa de Estancias Infantiles de la SEDESOL (tesis de maestría). México: Flacso.

Valencia, R.E. (2014). Necesidades de profesionalización para la detección de necesidades educativas especiales: responsables de la atención en Centros de Educación Inicial (tesis de maestría). Secretaría de Educación y Cultura. Hermosillo, Sonora.

Vernon-Feagans, L., Bratsch-Hines, M. E., \& the Family Life Project Key Investigators. (2013). Caregiver-Child Verbal Interactions in Child Care: A Buffer against Poor Language Outcomes when Maternal Language Input is less. Early Childhood Research Quarterly, 28(4), 858-873. 